\title{
Electron Microscopy as an Emerging Analytical Tool for Characterizing Biopharmaceuticals.
}

Bridget Carragher, Anette Schneemann, Joyce J. Sung, Sean K. Mulligan, Jeffrey A. Speir, Kat On, Joel Quispe, Clinton S. Potter

NanoImaging Services, Inc., San Diego, CA USA

Characterization of nanoparticles and biologics is a critical step in the development of important new pharmaceutical products and biosimilars. Biologics pose unique characterization challenges that require an interdisciplinary approach in which several orthogonal methods are used to provide a complete picture. The physical characteristics of a biological product include properties such as the size, shape, morphology and aggregation state of the particles. These properties are often dependent on the specific environment of the particles and thus ideally must be assessed under conditions that reflect the final formulation of the pharmaceutical. Electron microscopy (EM) and in particular cryo-electron microscopy (cryoEM), has a unique advantage in that it provides a direct means of observing the individual particles in a sample, preserved in their natural hydrated state (cryoEM), simultaneously providing information on homogeneity, size distribution, titer, morphology, preservation state, flexibility, and aggregation state. For particles with a regular size and shape, particle averaging methods can provide 3D structural information, complementing X-ray crystallography analysis.

We will demonstrate the use of EM as an analytical characterization tool by presenting a number of case studies as highlights. Specifically, we will discuss the characterization of Human Papilloma Virus (HPV) VLPs in GARDASIL ${ }^{\circledR}$, including the structure of the VLPs alone, on adjuvants, and when interacting with neutralizing antibodies [1]. We will also show how TEM was used as a non-intrusive tool to understand the structure and function of Hepatitis B surface antigen (rHBsAg) VLPs, the active component in the HBV vaccine [2]. We will furthermore demonstrate how TEM can be used to provide supporting information for characterization of a biosimilar drug delivery nanoparticle, a recombinant tuberculosis vaccine antigen, interacting with a lipid-based adjuvant [3], and a bi-specific, tetravalent immunoglobulin G-like molecule [4].

\section{References:}

[1] Zhao Q, et al. Characterization of virus-like particles in GARDASIL(R) by cryo transmission electron microscopy. Hum Vaccine Immunother. 10 (2013), 1.

[2] Mulder AM, et al. Toolbox for non-intrusive structural and functional analysis of recombinant VLP based vaccines: a case study with hepatitis B vaccine. PloS One. 7 (2012), 4.

[3] Fox CB, et al. Cryogenic transmission electron microscopy of recombinant tuberculosis vaccine antigen with anionic liposomes reveals formation of flattened liposomes. Int J Nanomedicine. 9 (2014); 1367.

[4] Correia I, et al. The structure of dual-variable-domain immunoglobulin molecules alone and bound to antigen. MAbs. 5 (2013), 364. 


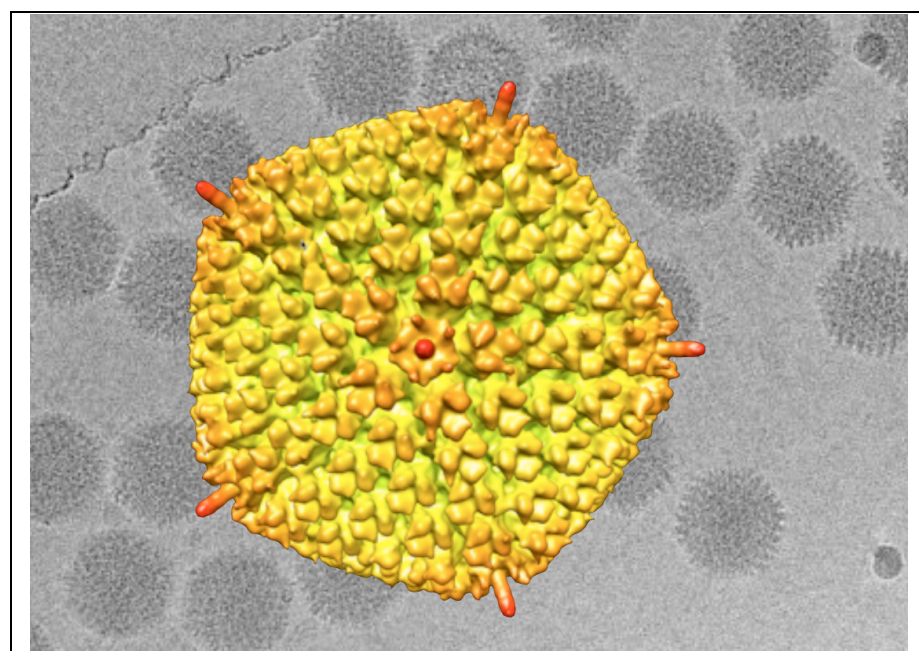

Figure 1: CryoEM of Adenovirus (background) and $3 \mathrm{D}$ reconstruction using single particle methods (foreground).

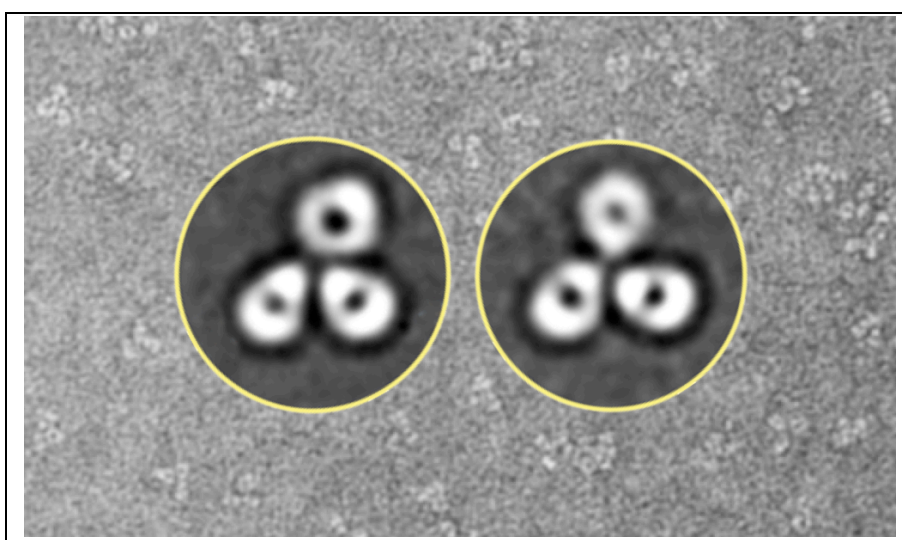

Figure 3: $\mathrm{EM}$ of Anti-HER2 $\mathrm{mAb}$ (background) and 2D class averages (foreground).

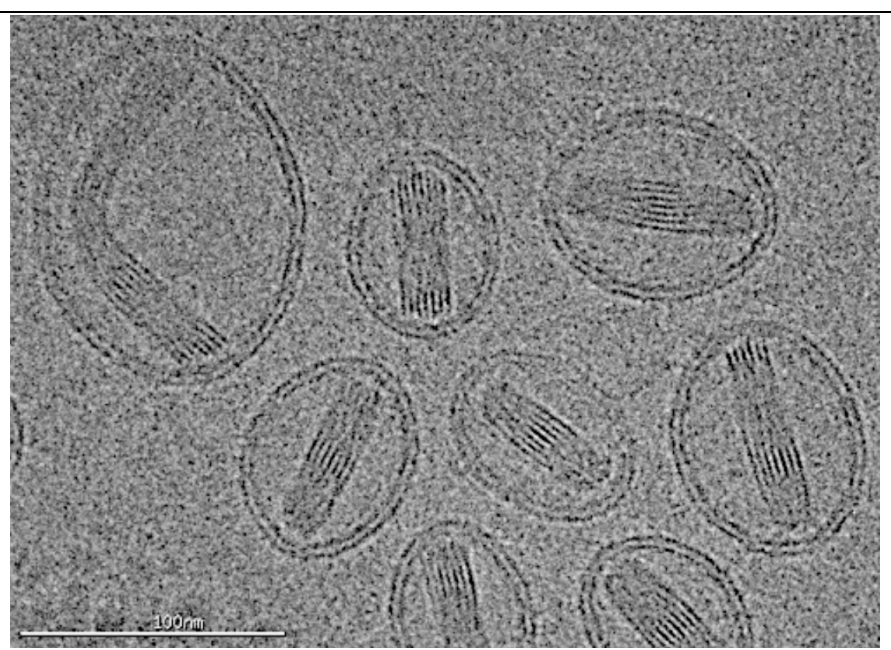

Figure 2: CryoEM of liposomal encapsulated doxorubicin.

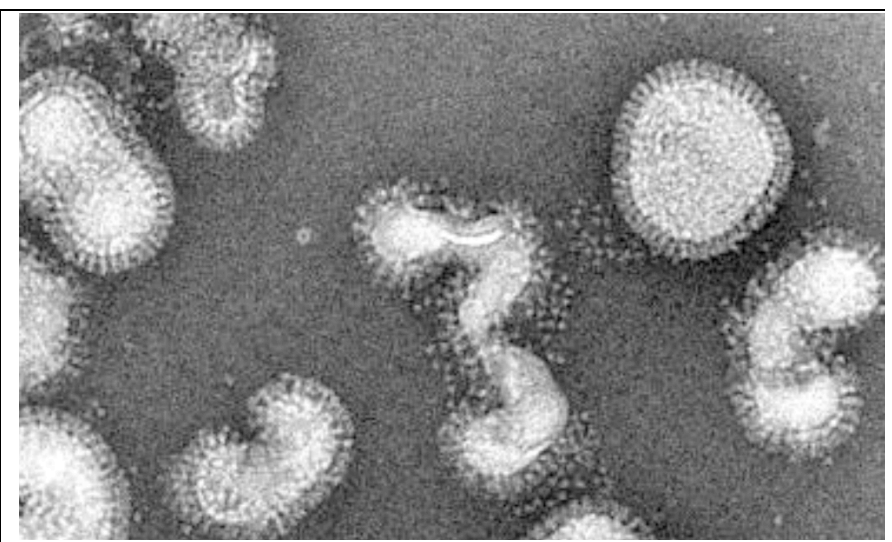

Figure 4: EM of H1N1 (influenza) vaccine. 